\title{
A Few Connectivity Technologies Involved in Internet of Things
}

\author{
Eswar Patnala ${ }^{1}$, Rednam S.S. Jyothi ${ }^{1}$, K. Asish Vardhan ${ }^{2}$, \\ N. Thirupathi Rao ${ }^{3}$, Debnath Bhattacharyya ${ }^{3}$ and Hye-jin Kim ${ }^{4}$ \\ ${ }^{1}$ Dept. of Information Technology, UCEV-JNTUK, INDIA. \\ ${ }^{2}$ Dept. of CS \& SE, College of Engineering., \\ Andhra University, India \\ ${ }^{3}$ Department of Computer Science and Engineering, \\ Vignan's Institute of Information Technology, \\ Visakhapatnam-530049, India \\ ${ }^{4}$ Business Administration Research Institute, Sungshin W. University, \\ 2, Bomun-ro 34da-gil, Seongbuk-gu, Seoul \\ (Corresponding Author) \\ eswar.patnala@gmail.com,ashi.mintu@gmail.com,nakkathiru@gmail.com, \\ debnathb@gmail.com, hyejinaa@daum.net
}

\begin{abstract}
Day by Day Smart technologies are playing vital role in our environment, industries and day to day life, as they are playing a key role. We should arrange them in such a way that their communication should be easy and flexible. For this purpose we have emerging technology named IOT (Internet of Things). In this technology various devices will communicate one another by using various connectivity protocols for better protocols.
\end{abstract}

Keywords: Sensor, actuator, zigbee, RFID, 6LowPAN

\section{Introduction}

IOT is the one of the emerging technology for effective development of smart homes and smart cities. In IOT, various types of devices, machines are connected by using wireless technology to the internet. As per the statistics, more than 9 billion things are connected to internet, using IOT. By using IOT, we can combine various types of technologies, such as low power embedded systems. Cloud computing, big data, machine learning and networks.

\subsection{Background History on the IOT Terminology}

After entering into the millennium, the current generation is entering into the new era of technology, where users using the internet will be in more number and where the humans may become minority as generators and receivers of traffic. The most of the traffic will flow between the device and all kinds of things. There by introducing a technology called Internet of Things (IOT).

There are various areas of IOT enablers, they are

a) RFID

b) Nano technology

c) Sensors

d) Smart Networks

Received (May 27, 2017), Review Result (August 15, 2017), Accepted (September 11, 2017) 


\subsection{Definition}

The Internet of Things (IOT) is the internetworking of physical devices, vehicles (also referred to as "connected devices" and smart "devices"), buildings and other items embedded with electronics, software, actuators and network connectivity which enable these objects to collect and exchange data.

\subsection{Characteristics of IOT}

Efficient, scalable, unambiguous naming and internet connectivity are important characteristics of IOT. Because of these characteristics, IOT has been spreaded across different sectors such as manufacturing of business, health care, retail and security. Among all these manufacturing of business occupies major share in using IOT.

\subsection{Connectivity Terminologies}

We use various connectivity methods for the interconnection of things. For short range communication, we use LAN's and for Geographical wide communication, we use WAN's and for better communication inside the LAN, we install nodes and for routing the packets between LAN and WAN on the IP layer, we Gateways and proxy is used to perform active application layer functions between IOT nodes and other entities.

\section{Sensing}

While building IOT, the most important elements are sensors and actuators. First the sensor senses the data and then that is passed to actuator through the network to perform respective action.

\subsection{Definition of Sensor}

A sensor detects (senses) changes in the ambient condition or in the state of another device or a system, and forwards or processes this information in a certain manner. The sensor device senses the environmental characteristics and gives the response according to the input [1]. For example, heat is converted to electrical signals in temperature sensor and atmospheric pressure is converted to electrical signal in barometer [1].

\subsection{Transducers}

The main purpose of transducers is to change the energy of one kind to another. For example, in sound system, microphone acts as $\mathrm{i} / \mathrm{p}$ device which converts into electrical signals for an amplifier to amplify a process and output device i.e., loud speaker converts these electrical signals into sound waves [2].

\subsection{Classes of Sensors}

Sensors are divided into classes based on output and data type. Based on output, the sensors are divided into analog and digital. Now in data type, the sensors are divided into scalar and vectors.

\section{Sensor Types:}

There are various types sensor. They are 


\subsubsection{Light Sensor}

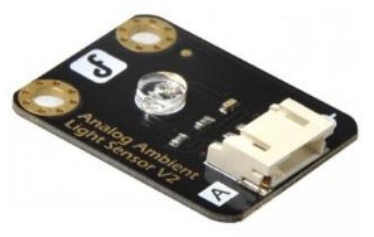

Figure 1. Light Sensor

The working of this particular sensor works on the light that was being generated from any other sources of light. The main use of this sensor device is to find or calculate the intensity of the light that was being generated or observed.

\subsubsection{Temperature Sensor:}

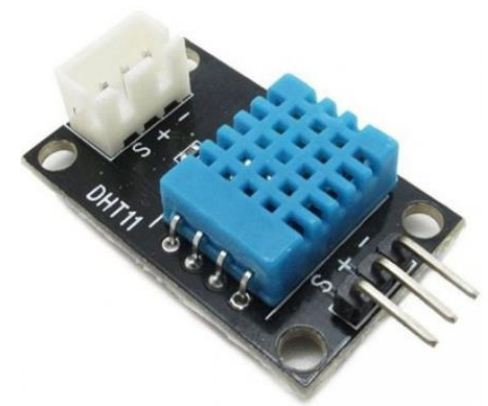

Figure 2. Temperature Sensor

This sensor device is mainly used for the purpose of measuring or calculating the temperature value that was identified at the places where this device is deployed. The main usage is to measure or calculate the temperature values at various places.

\subsubsection{Force Sensor:}

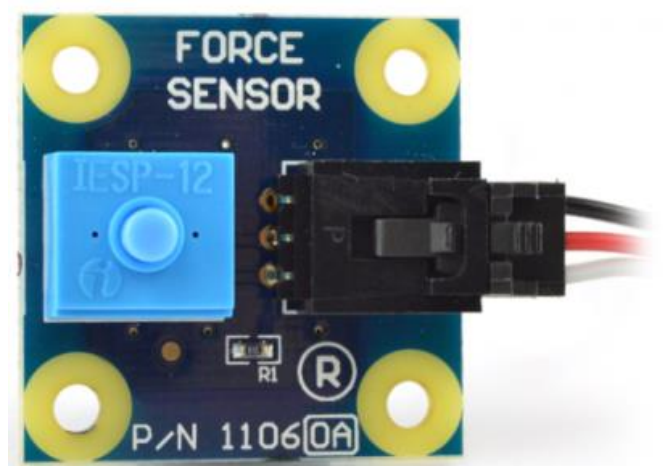

Figure 3. Force Sensor

The current force sensor is used in various vehicles like cars, two wheelers and other heavy vehicles. The main utility if this sensor unit was it measures the force or the energy that was being generated through the movement of the vehicles. 


\subsubsection{Position Sensor:}

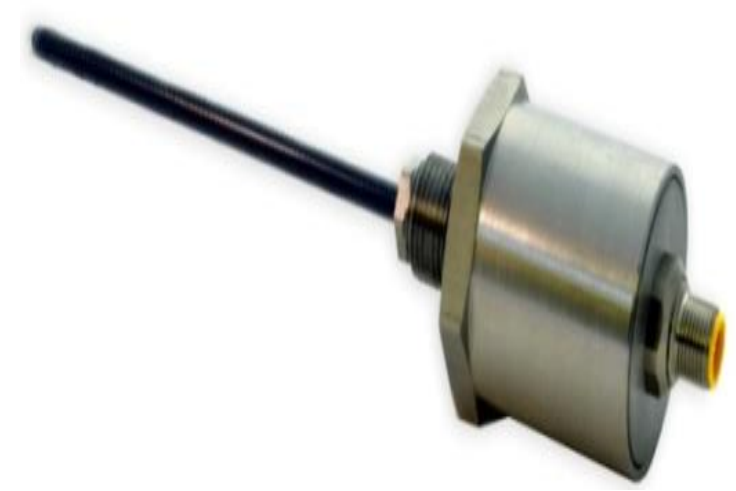

Figure 4. Position Sensor

The main work of the current sensor device is to measure the position of the various devices or the vehicles. It can be used to measure or identify the position of various vehicles.

\subsubsection{Speed Sensor:}

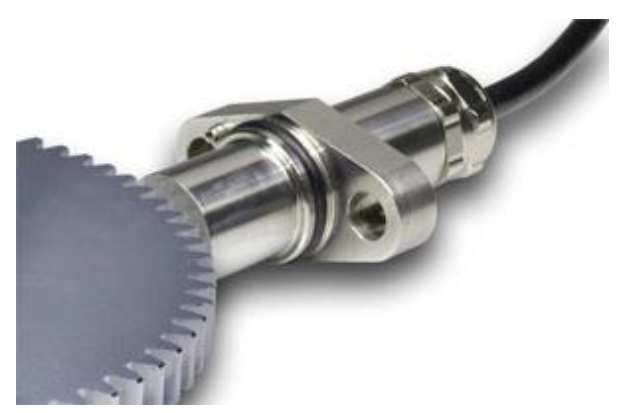

Figure 5. Speed Sensor

\subsubsection{Chemical Sensor:}

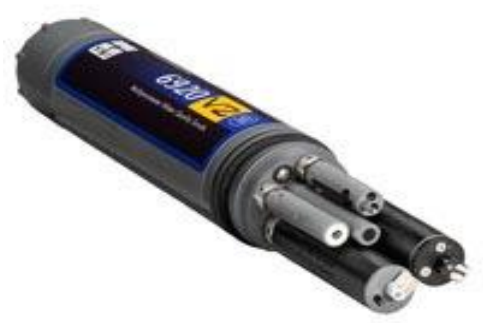

Figure 6. Chemical Sensor

\section{Actuation}

In Actuation process, we use actuators to control the mechanisms or the systems. The response of actuators depends upon the environment and actuator requires a control signal and source of energy as inputs .On receiving the control signal. The actuator responds by converting the energy into mechanical motion. The control system might be software based or human [9]. 


\subsection{Actuator types:}

There are various types actuators with respective to the sensors, the different types of actuators available are

- Hydraulic Actuator

- Pneumatic Actuator

- Mechanical Actuator

- Thermal Actuator

- Electrical Actuator

\section{Connectivity Technologies}

In order to provide communication between sensor and actuators, we have various number of communication protocols.

They are:

- IEEE 802.15.4

- Zigbee

- 6LowPAN

- RFID

- Bluetooth

\subsection{IEEE 802.15.4:}

It is the standard used for low data-rate wireless Personal Area Networks. And it is used for developing the low power consumption and low data rate application specifically for monitoring and control application. Phy, MAC, LLC (logic link control) layers are used by IEEE 802.15 .4 standard.

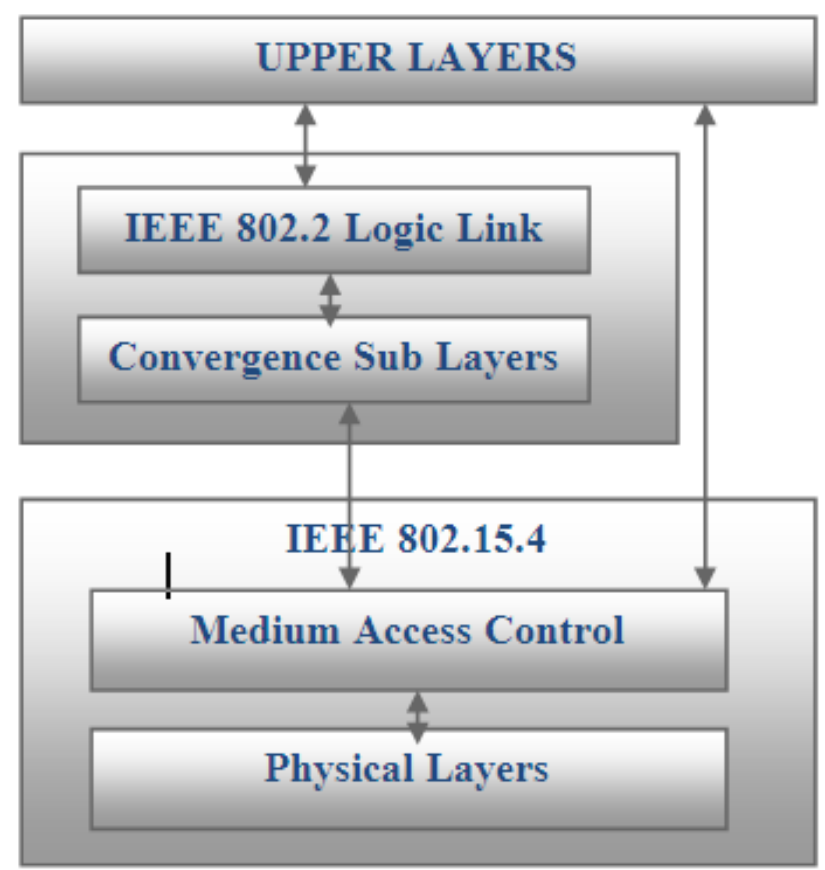

Figure 7. IEEE 802.15.4 Protocol Stack

In order to communicate with upper layers it uses service specific convergence layer. The topologies used by this standard are star and Mesh [11]. 
There are two types of IEEE 802.15.4 networks, they are

i. Non-Beacon Enabled

ii. Beacon Enabled

\subsection{Zigbee}

Zigbee was the enhanced version of IEEE 802.15.4.it was the designed by layer 3 and above. These layers collaboratively works with layer 2.To avail additional features in communication, layer 4 provide its services with layer 3 .

Authentication of node encryption for security and data routing \& forwarding capability that enables mesh networking are the extra features which are provided by the Zigbee other than IEEE 802.15.4 protocol [11].

The most eminent use of zigbee is wireless sensor networks using mesh topology.

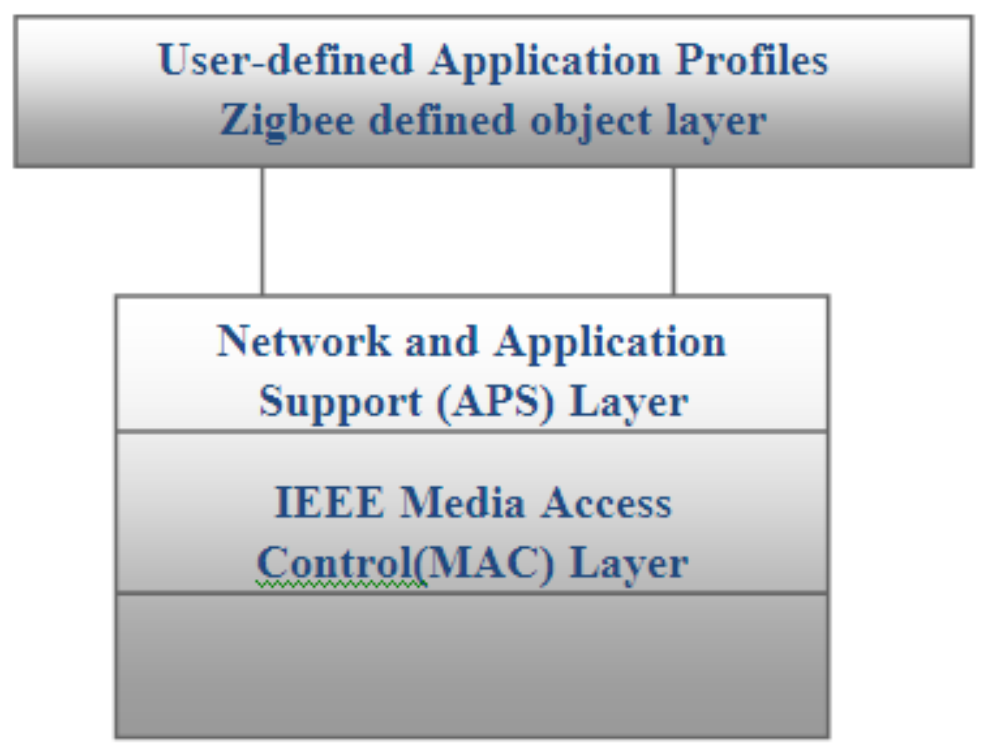

Figure 8. Zigbee Architecture

\subsubsection{Components in Zigbee}

In order implement some operations by zigbee, this protocol includes two components

\subsubsection{Zigbee Device objects (ZDO):}

The features like device management, security, policies are the service provided by the Zigbee device management.

\subsubsection{Application Support sub-layer (APS):}

The main service provided by the APS is to acts as bridge between the network and other layers and it also provides the interfacing and control services. 


\subsubsection{Zigbee Topologies}

\subsubsection{Star Topology}

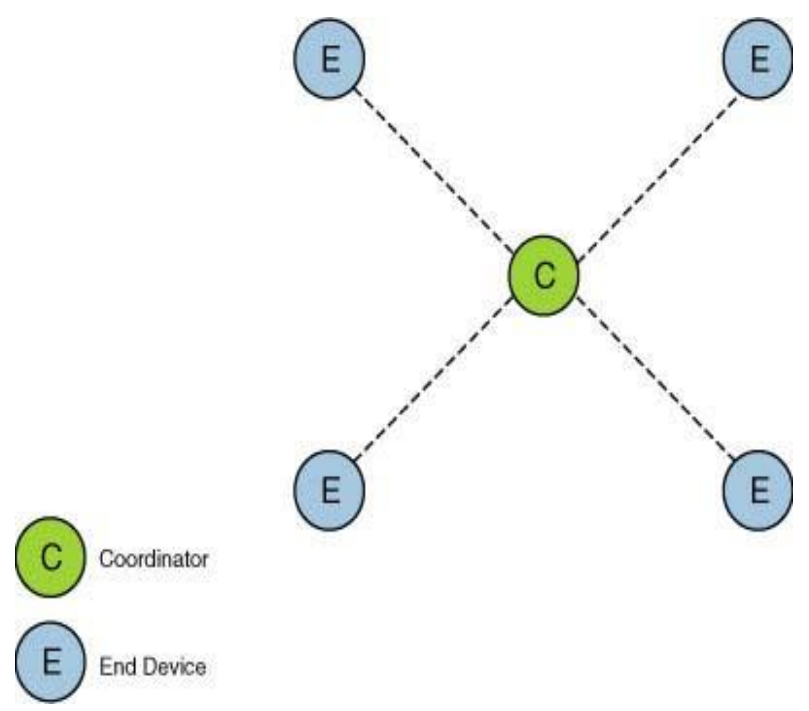

Figure 9. Star Topology

\subsubsection{Mesh Topology}

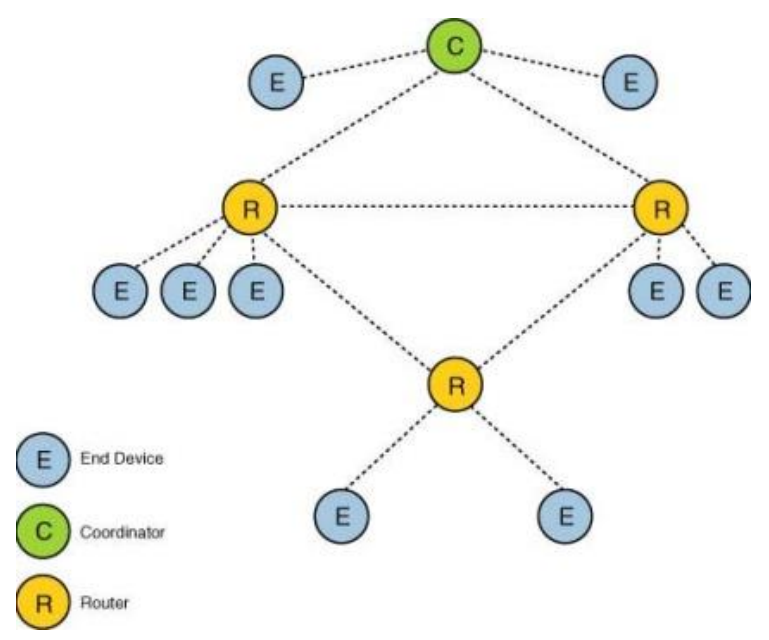

Figure 10. Mesh Topology

\subsubsection{Zigbee Mesh}

Among all the topologies, mesh was the most reliable one. In this topology, a node can communicate with any other node within its range.

If a node wants to send the data to the node which was not within its range then the source node passes the data through several intermediate nodes to reach the data. If any node was failed then by using other nodes, it can still transmit the data. The main advantage of mesh networks are, they can configure themselves and they can self heal [11]. 


\subsubsection{Types of Zigbee}

\subsubsection{Zigbee Co-coordinators}

For each network, there will be single Zigbee coordinator and the coordinator is responsible for forming the root of zigbee network tree and acts as bridge between networks. It also stores information regarding the network and acts as a trust centre \& repository for security keys.

\subsubsection{Zigbee Router}

The main function of the router is to run the application and another function is to relay the information between nodes connected to it.

\subsubsection{Zigbee End Device (ZEO)}

When compared to other types, it has very limited functionality and it can only communicate with parent node. If the end device has any work, it will be wake up state and otherwise it goes to the sleep state.

\section{Application:}

- Telecommunication

- Home automation

- Health care

\subsection{LowPAN}

\subsubsection{Introduction}

Low-Power Wireless Personal Area Network over IPV6 (6LowPAN).Smallest devices with limited capability uses this protocol. Though the devices have low power but they can still connect to the internet by this protocol.

\subsubsection{Features of 6LowPAN}

It allows IETF 802.15.4 to carry 128-bit address of IPV6. 6LowPAN protocol is used for developing IOT and smart grid application.

\subsubsection{Addressing in 6LowPAN}

Addressing in 6lowPAN are divided into two types they are

- 64bit extended

- 16 bit short

IPV6 packets are transmitted as link layer broadcast frames.16 bit address was allocated by PAN coordinator which was PAN specific.64 bit extended address by globally unique.

Routing Protocols used by 6LowPAN protocol are

- LOADng

- RPL

\subsubsection{LOADng}

The operation of LOADng routing protocol is used to generate Route Request(RREQs) and Route replies(RREPs).route Request are generated by LOADng router and forwards 
them until they reach destination. After receiving the RREQ by corresponding destination, RREPs are sent by destination to source using unicast mechanism [17].

\subsubsection{RPL Routing}

It is a distance vector IPV6 routing protocol for lossy and low power networks. In this data is transmitted in the form of datagram's and they include the routing information. It contains two mechanisms. They are

$$
\text { - Proactive }
$$

- Reactive

Proactive: It maintains Routing Topology and information related to it.

Reactive: Resolving routing inconsistencies, message confidentiality and integrity are the services provided by RPL. It supports data path validation and node detection.

\subsection{RFID}

RFID is an acronym for "radio frequency identification". In this RFID mechanism, information will be encoded and store in RFID tags and this information will be displayed on the screen, when they are read by reader. This mechanism was similar to the barcodes. FID was the advanced mechanism of Barcodes and QR codes[18].

\subsubsection{Features}

The components involved in RFID are integrated circuit and an antenna. The RFID tag was covered by protection material. So that it can be guarded from environmental effects.

There are two types of tags. They are

- Passive

- Active

\subsubsection{Passive}

Passive tags do not contain any battery and the power supply for passive tags is supplied through the reader [18].

\subsubsection{Active}

Components present in active tags are transmitters and the power source i.e., in the form of batteries [18].

\subsubsection{Application}

- Inventory management

- ID cards

- Pharmaceutical Industry

\section{Conclusion}

We have various types of sensors used in IOT and actuators in the above sections. In order to provide communication between these sensors and actuators. We have discussed some the connectivity protocols and their working procedure. 


\section{References}

[1] http://www.businessdictionary.com/definition/sensor.html,https://en.oxforddictionaries.com/definition/s ensor

[2] http://www.robotshop.com/en/gravity-ambient-light-sensor.html

[3] http://www.robotshop.com/en/electronic-brick-humidity-temperature-sensor.html

[4] http://www.robotshop.com/en/phidgets-force-sensor.html

[5] http://alliancesensors.com/linear-position-sensor-mr-7-series.

[6] http://www.sensorprod.com/lenord/speed-sensor-gel-2478.php

[7] http://www.lakescientist.com/chemical-sensors/Source: L.Fenzel, "What's The

[8] Difference Between IEEE 802.15.4 And ZigBee Wireless?”, Electronic Design (Online), (2013).

[9] http://www.tutorial-reports.com/wireless/zigbee/zigbee-architecture.php).

[10] http://ptgmedia.pearsoncmg.com/images/chap2_9780137134854/elementLinks/02fig02.jpg.

[11] http://ptgmedia.pearsoncmg.com/images/chap2_9780137134854/elementLinks/02fig05.jpg

[12] Wireless Sensor Networks Research Group". Sensor-networks.org. 2010-04-15.

[13] Source: L.Fenzel, "What's The Difference Between IEEE 802.15.4 And ZigBee

[14] Wireless?”, Electronic Design (Online), (2013)

[15] "How does RFID work?" AB\&R (Online) 